\title{
Antibacterial activity of neem (Azadirachta indica) leaves on Vibrio spp. isolated from cultured shrimp.
}

\begin{abstract}
The use of antibiotics in aquaculture to treat infections has resulted in the development of resistant strains which have rendered antibiotic treatment ineffective. Therefore, alternative antibacterial materials must be found. Extracts of neem tree (Azadirachta indica) leaves were tested against Vibrio parahaemolyticus and Vibrio alginolyticus isolated from cultured shrimp. Aqueous extract of neem leaves did not produce any inhibitory zone while the neem juice produced inhibitory zone that showed linear relationship to the concentration of neem juice on both bacteria. The Minimum Inhibitory Concentration (MIC) for V. parahaemolyticus and V. alginolyticus was 3.13 and $6.25 \%$, respectively. The Minimum Bactericidal Concentration (MBC) for V. parahaemolyticus and V. alginolyticus was 12.50 and $25.00 \%$, respectively. It is concluded that neem juice is an antibacterial agent and is useful for inhibition of vibrios in shrimp.
\end{abstract}

Keyword: Antimicrobial sensitivity test; Azadirachta indica; Minimum bactericidal concentration; Minimum inhibitory concentration; Neem; Vibrio spp. 